\title{
FLUCTUACIÓN POBLACIONAL, PLANTAS HUÉSPEDES, DISTRIBUCIÓN Y CLAVE PARA LA IDENTIFICACIÓN DE PLATYPODINAE (COLEOPTERA: CURCULIONIDAE) ASOCIADOS AL AGROECOSISTEMA CACAO EN TABASCO, MÉXICO
}

\author{
Manuel Pérez de la Cruz, ${ }^{1,4}$ Jorge M. Valdéz Carrasco, ${ }^{2}$ Jesús Romero \\ Nápoles, ${ }^{2}$ Armando Equihua Martínez, ${ }^{2}$ Saúl Sánchez $\operatorname{Soto}^{3} \&$ Aracely de la \\ Cruz Pérez ${ }^{1}$ \\ ${ }^{1}$ Universidad Juárez Autónoma de Tabasco, División Académica de Ciencias Biológicas, Código \\ Postal 86150, Carretera Villahermosa-Cárdenas km $0.5 \mathrm{~s} / \mathrm{n}$ entronque a Bosque de Saloya, \\ Villahermosa, Tabasco, México. \\ ${ }^{2}$ Colegio de Postgraduados, Campus Montecillo, Apartado Postal 56230 Km. 36.5 Carretera México- \\ Texcoco, Montecillo, Texcoco, Estado de México, México. \\ ${ }^{3}$ Colegio de Postgraduados, Campus Tabasco, Apartado Postal 24 86500, H. Cárdenas Tabasco, \\ México. \\ ${ }^{4}$ Correspondencia, E-mail: perezmandoc@hotmail.mx, perezman@colpos.mx
}

Pérez de la Cruz, M., J. M. Valdéz Carrasco, J. Romero Nápoles, A. Equihua Martínez, S. Sánchez

Soto \& A. de la Cruz Pérez. 2011. Fluctuación poblacional, plantas huéspedes, distribución y clave para la identificación de Platypodinae (Coleoptera: Curculionidae) asociados al agroecosistema cacao en Tabasco, México. Acta Zool. Mex. (n. s.), 27(1): 129-143.

RESUMEN. Se estudió la fluctuación poblacional, huéspedes y distribución de platipódidos asociados al agroecosistema cacao en Tabasco, México durante el año 2007. Los insectos fueron capturados con trampas de alcohol, luz y en sus plantas huéspedes en cuatro localidades. Se recolectaron cinco especies siendo Teloplatypus excisus (Chapuis) el que presentó la mayor abundancia con un total de 346 especímenes. T. excisus y Tesserocerus dewalquei Chapuis son nuevos registros para Tabasco. Se encontró a Spondias mombin como nuevo huésped de Platyscapulus pulchellus (Chapuis) y T. excisus. El método de captura más eficiente fue la trampa de alcohol, con la cual se capturo 459 especímenes. La fluctuación poblacional de las especies a través del año de estudio se mantuvo en niveles bajos en la mayoría de las localidades estudiadas a excepción de Teapa, donde se registraron picos poblacionales marcados en mayo y julio tanto en las trampas de alcohol como directamente en sus hospederos y en los meses de julio y noviembre en las de luz.

Palabras clave: insectos, agroecosistema, abundancia, escarabajos, guía. 
Pérez de la Cruz, M., J. M. Valdéz Carrasco, J. Romero Nápoles, A. Equihua Martínez, S. Sánchez Soto \& A. de la Cruz Pérez. 2011. Dynamic population, hosts plants, distribution and identification key for Platypodinae (Coleoptera: Curculionidae) associated with the cacao agroecosystem in Tabasco, Mexico. Acta Zool. Mex. (n. s.), 27(1): 129-143.

ABSTRACT. The ambrosia beetle dynamic population, hosts and distribution were studied in the cacao agroecosystem in Tabasco, Mexico during 2007. The insects were collected in four localities with ethanol and light traps and by direct collecting in their host plants. Five species were collected with Teloplatypus excisus (Chapuis) being the most abundant with a total of 346 specimens. T. excisus and Tesserocerus dewalquei Chapuis are new records for Tabasco. Spondias mombin is recorded as a new host to Platyscapulus pulchellus (Chapuis) and T. excisus. The most efficient method of captured was the ethanol trap where which 459 specimens captured. The populational dinamic of the species through the year of study stayed in low levels in most of the towns studied, with exception of Teapa where market populational pick was seen in May and July through use the ethanol traps and in its hosts; and in the months of July and November with the light traps.

Key Words: insect, agroecosystem, abundance, beetle, key.

\section{INTRODUCCIÓN}

Las especies de Platypodinae son conocidos comúnmente como escarabajos de ambrosia ya que la mayoría de las especies son xilomicetófagas (se alimentan de hongos ectosimbióticos), aunque algunas de ellas son xilófagas (se alimentan de madera), Los platipódidos ambrosiales barrenan la madera de sus hospederos, construyen sus galerías e introducen los hongos con los cuales están asociados, éstos constituirán la fuente principal de alimento para las larvas y adultos. Estos insectos generalmente atacan árboles debilitados o recién muertos, aunque existen algunas especies capaces de matar plantas sanas (tal es el caso de Euplatypus segnis importante como plaga primaria del nogal, ya que sus infestaciones causan la muerte del árbol y reducen la producción de nuez según Cibrián et al. (1995)); La mayoría de las especies de este grupo presentan cierta especificidad por una especie o género de huésped (Schedlarius mexicanus (Duges) solamente ha sido recolectado en especies del género Burse$r a$ ), otras pueden establecerse y reproducirse en varios huéspedes (E. parallelus ataca una gran variedad de arboles en zonas tropicales reduciendo su valor comercial en las especies maderables según Cibrián et al. (1995)). La mayor parte de las especies de platipódidos se distribuyen en zonas tropicales (Arnett 1973) y los trabajos que se han realizado en México han sido desde el punto de vista taxonómicos con datos puntuales de recolecta (Burgos-Solorio \& Equihua 2007, Atkinson \& Equihua 1988, Atkinson \& Equihua 1985, Equihua \& Atkinson 1987, Equihua 1985, Equihua et al. 1984, Schedl 1940).

El objetivo de este estudio es dar a conocer información sobre los platipódidos asociados al agroecosistema cacao en Tabasco, México; así como, proporcionar una clave para facilitar la identificación de estos insectos. 


\section{MATERIAL Y MÉTODOS}

El estudio se realizó de enero a diciembre del año 2007 en cuatro plantaciones de cacao del estado de Tabasco. En la primera plantación se utilizó un predio de seis hectáreas de 37 años de edad, sembradas a una distancia de $4 \times 4 \mathrm{~m}$, la cual se localiza entre las coordenadas $17^{\circ} 59^{\prime} 23^{\prime \prime}$ de latitud norte y 9337'09” de longitud oeste, de $20 \mathrm{msnm}$ en el Ejido Las Delicias de la Ranchería José María Morelos y Pavón, municipio de Teapa, Tabasco, México (Teapa). La fisiografía de la zona corresponde a un valle abierto, con un clima cálido húmedo con lluvias todo el año (Af), la temperatura promedio es de $26^{\circ} \mathrm{C}$. La precipitación alcanza una media anual de 3424 mm (Instituto Nacional de Estadística Geografía e Informática (INEGI) 2006). La segunda plantación corresponde a una hectárea de aproximadamente 38 años de edad, localizada entre las coordenadas $17^{\circ} 37^{\prime} 19^{\prime \prime}$ de latitud norte y $92^{\circ} 27^{\prime} 56^{\prime \prime}$ de longitud oeste, en el kilómetro 21 de la carretera Cárdenas, Tabasco-Coatzacoalcos, Veracruz, México ( $\mathrm{km} 21)$. La tercera plantación consta de dos hectáreas de 10 años de edad, localizadas entre las coordenadas $17^{\circ} 58^{\prime} 09^{\prime \prime}$ de latitud norte y $93^{\circ} 20^{\prime} 55^{\prime \prime}$ de longitud oeste en el Ejido El Bajío 2a sección, municipio de Cárdenas, Tabasco (El Bajío). La cuarta plantación tuvo una extensión de 3.3 hectáreas con 45 años de edad localizada entre las coordenadas $18^{\circ} 02^{\prime} 34^{\prime \prime}$ de latitud norte y $93^{\circ} 22^{\prime} 44^{\prime \prime}$ de longitud oeste en el Ejido Río Seco $2^{\mathrm{a}}$ sección, municipio de Cárdenas, Tabasco (Río Seco). Las tres últimas plantaciones se encuentran a $10 \mathrm{msnm}$, sembradas a una distancia de $4 \mathrm{x} 4 \mathrm{~m}$. Su fisiografía corresponde a la de llanura costera del Golfo Sur, con clima cálido húmedo con abundantes lluvias en verano (Am), la temperatura promedio es de $26.4^{\circ} \mathrm{C}$. La precipitación media anual es de 1994 mm (INEGI 2005).

Captura de platipódidos mediante trampas con alcohol etílico. El tipo de trampa de alcohol utilizado consta de un embudo de plástico, en cuyo extremo inferior posee un recipiente para recolectar los insectos y en la parte superior una pantalla de plástico transparente, por encima de la cual se adicionó una tapa de plástico. Dentro de la pantalla se colocó un tubo de plástico con diámetro de $15 \mathrm{~mm}$, en el cual se agregó alcohol etílico desnaturalizado con octa-acetato de sacarosa marca Protec al $0.03 \mathrm{~g}$ de uso comercial al 70\%, como material atrayente. Se instalaron cinco trampas en una hectárea ubicada en el centro de cada plantación, a una altura de $1.50 \mathrm{~m}$, a $50 \mathrm{~m}$ de distancia entre ellas formando un cuadrado con una trampa en el centro. La recolección de los insectos atraídos en cada una de las trampas se realizó quincenalmente, los especímenes se conservaron en alcohol al 70\% para su posterior determinación. En cada recolección se procedió a reponer el atrayente (Bustamante \& Atkinson 1984, Iturre \& Darchuck 1996).

Captura de platipódidos con trampa de luz fluorescente. Se utilizó una trampa similar a la de alcohol antes descrita para recolectar a los platipódidos sustituyendo el material atrayente por una fuente de luz fluorescente proporcionada por una lámpara recargable marca Lloyd's de 20 watts con una duración aproximada de 4 horas, colo- 
cada ésta a un costado de la trampa. La trampa se instaló en el centro de la plantación en cada una de las localidades de estudio a una altura de $1.50 \mathrm{~m}$ a las 18:00 horas y fue levantada al día siguiente; los muestreos se realizaron mensualmente. Los especímenes se conservaron en alcohol etílico al 70\% para su posterior determinación.

Captura directa de platipódidos sobre sus plantas huéspedes. Se aplicó el método de la captura directa sobre las plantas huéspedes, considerando que el ciclo de estos barrenadores transcurre dentro de ellas. La recolecta consistió en hacer revisiones directamente sobre las partes de las plantas (tallo, ramas y frutos) en donde se observo la presencia de estos insectos. La presencia de aserrín, huecos de entrada y grumos de madera fueron de gran utilidad en el campo para detectar el ataque de estos barrenadores (Bustamante \& Atkinson 1984). Los muestreos se realizaron quincenalmente durante el año de estudio. El material vegetal fue llevado al laboratorio y colocado en cámaras de emergencia para la posterior recolección de los insectos. Los organismos recolectados se conservaron en alcohol etílico al $70 \%$ para su posterior identificación.

Identificación del material biológico. La determinación taxonómica de los organismos se llevó a cabo mediante el uso de claves taxonómicas a nivel de género (Wood 1993) y a nivel de especies mediante comparaciones con material depositado en la colección del Colegio de Postgraduados Campus Montecillo, México (CEAM). Para obtener los datos de distribución, nombres válidos y registros anteriores para el estado de Tabasco se consultaron las siguientes publicaciones: Sharp \& Blandford (1895-1907), Schedl (1940), Atkinson \& Equihua (1985), Atkinson \& Equihua (1988), Equihua et al. (1984), Equihua (1985), Equihua \& Atkinson (1987), Wood \& Bright (1992a, 1992b), Bright \& Skidmore, (1997, 2002). Las fotografías fueron tomadas con un microscopio Carl Zeiss Tessovar, con cámara digital para microscopía Paxcam3. El procesamiento de las fotografías digitales se realizo con el programa combineZM y con Adobe Fotoshop CS3.

\section{RESULTADOS}

Se recolectaron cinco especies de cuatro géneros de platipódidos con un total de 514 especímenes con los tres métodos de captura utilizados, Teloplatypus excisus fue la especie más abundante con 346 individuos (67\% del total). Teapa fue la localidad donde se registró la mayor abundancia de estos insectos con 243 especímenes (47\%) con respecto al total, seguido del $\mathrm{km} 21$ con 121 (24\%), Río seco con 99 (19\%) y el Bajío presentó la menor con 51 (10\%). La especie que presentó la mayor abundancia en los cuatro sitios estudiados fue $T$. excisus, siendo Teapa en donde se registró la mayor abundancia con 159 organismos, lo que representa el $46 \%$ del total recolectado para esta especie, seguido del km 21 con 78 (23\%), Río seco con 72 (21\%) y El Bajío con 37 (11\%). Las especies T. excisus y Tesserocerus dewalquei son nuevos registros para Tabasco. Se encontró a Spondias mombin como nuevo huésped de Platyscapu- 
lus pulchellus y T. excisus los cuales compiten por el recurso en el agroecosistema cacao. El método de captura más eficiente fue la trampa de alcohol con la cual se obtuvo el mayor número de especímenes con 459, seguido de la trampa de luz con 46, en tanto que la captura directa sobre sus plantas huéspedes aportó el menor número de especímenes con nueve organismos (Cuadros 1 y 2).

La fluctuación poblacional de las especies de platipódidos recolectados a través del año de estudio en cada una de las localidades sumando los tres métodos de captura se presenta en la Figura 1 en ella se puede observar que las poblaciones tienen un comportamiento similar en la mayoría de los sitos ( $\mathrm{km}$ 21, El Bajío y Río seco), sin picos poblacionales marcados; a diferencia de Teapa, donde se pueden observar dos picos poblacionales marcados uno en mayo y el otro en julio. Algo similar se encontró al analizar por separado la fluctuación poblacional de estos insectos capturados sólo en las trampas de alcohol (Figura 2).

Los platipódidos recolectados en las trampas de luz en cada una de las localidades presentaron un comportamiento similar en el km 21, El Bajío y Río seco con poblaciones bajas a través del año de estudio; a diferencia de Teapa donde se pudo observar picos poblacionales marcados en febrero, julio y noviembre (Figura 3 ).

Cuadro 1. Especies y abundancia de platipódidos capturados con tres métodos de recolecta en el agroecosistema cacao en Tabasco, México.

\begin{tabular}{lcccrr}
\hline \multicolumn{1}{c}{ Especies } & T. de alcohol & T. de luz & Huéspedes & Total & \multicolumn{1}{c}{$\%$} \\
\hline Euplatypus parallelus (Fabricius) & 15 & 27 & 6 & 48 & 9.33 \\
Euplatypus segnis (Chapuis) & 101 & 6 & 0 & 107 & 20.81 \\
Platyscapulus pulchellus (Chapuis) & 0 & 5 & 2 & 7 & 1.36 \\
Teloplatypus excisus (Chapuis) & 342 & 3 & 1 & 346 & 67.31 \\
Tesserocerus dewalquei Chapuis & 1 & 5 & 0 & 6 & 1.16 \\
Total & 459 & 46 & 9 & 514 & 100 \\
\hline
\end{tabular}

Cuadro 2. Especie y abundancia de platipódidos capturados en las diferentes localidades de estudio en el agroecosistema cacao en Tabasco, México.

\begin{tabular}{lrrrr}
\hline \multicolumn{1}{c}{ Especies } & Teapa & $\mathrm{km} \mathrm{21}$ & El Bajío & Río seco \\
\hline Euplatypus parallelus & 25 & 10 & 9 & 4 \\
Euplatypus segnis & 48 & 31 & 5 & 23 \\
Platyscapulus pulchellus & 7 & 0 & 0 & 0 \\
Teloplatypus excisus & 159 & 78 & 37 & 72 \\
Tesserocerus dewalquei & 4 & 2 & 0 & 0 \\
Total & 243 & 121 & 51 & 99 \\
\hline
\end{tabular}




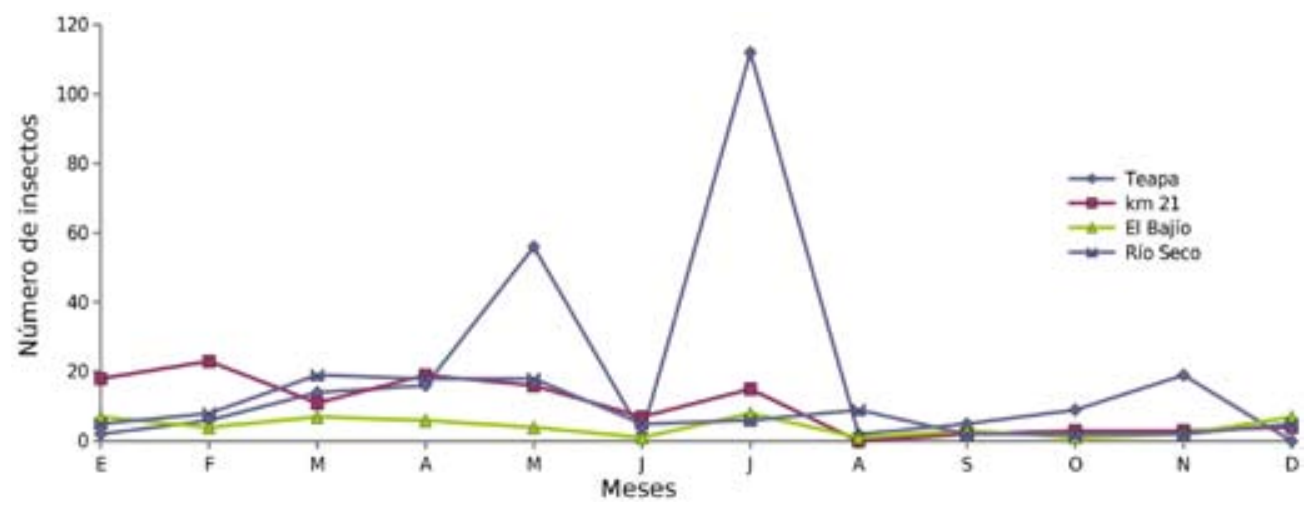

Figura 1. Fluctuación poblacional de platipódidos recolectados en cada una de las localidades estudiadas sumando la captura de los tres métodos de colecta.

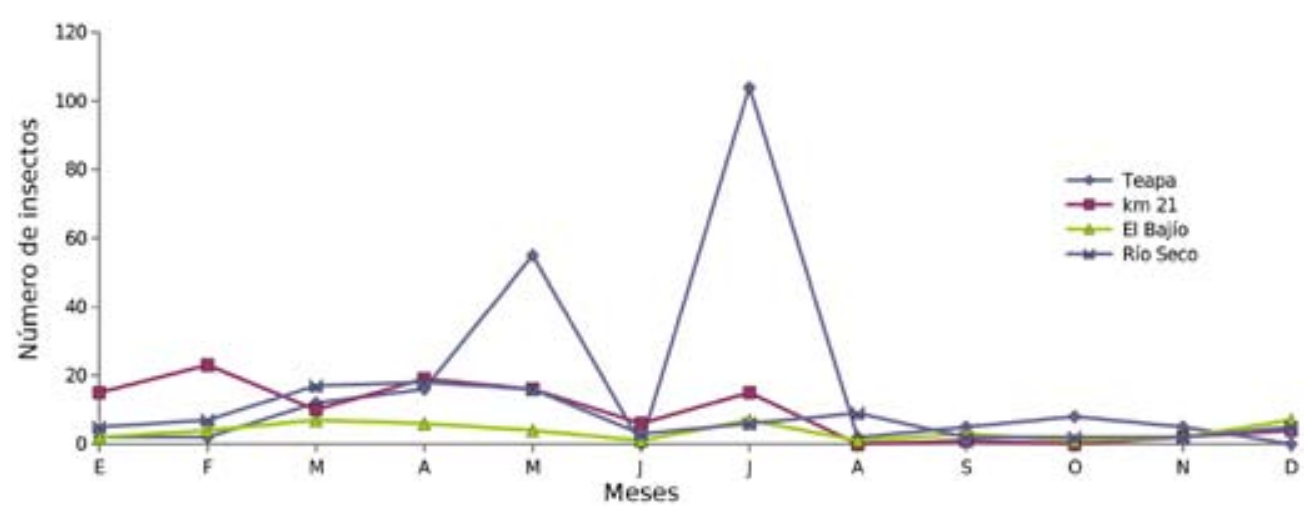

Figura 2. Fluctuación poblacional de platipódidos recolectados en cada una de las localidades utilizando las trampas de alcohol.

En la Figura 4 se presenta la fluctuación poblacional de las especies de platipódidos recolectados con los tres métodos de captura donde se muestran picos poblacionales marcados de T. excisus en mayo y en julio y de Euplatypus segnis (Chapuis) en mayo a diferencia de las otras especies que sus poblaciones se mantienen bajas durante el año. De manera general la fluctuación poblacional de los platipódidos independientemente del método de recolecta, registro picos poblacionales marcados en el mes de julio en la mayoría de los casos, coincidiendo con el inico de la temporada de lluvia y altas temperaturas, a diferencia de la trampa de luz en Teapa donde su máximo pico poblacional fue observado en noviembre después de un periodo de alta precipitación (Figuras 5 y 6). 


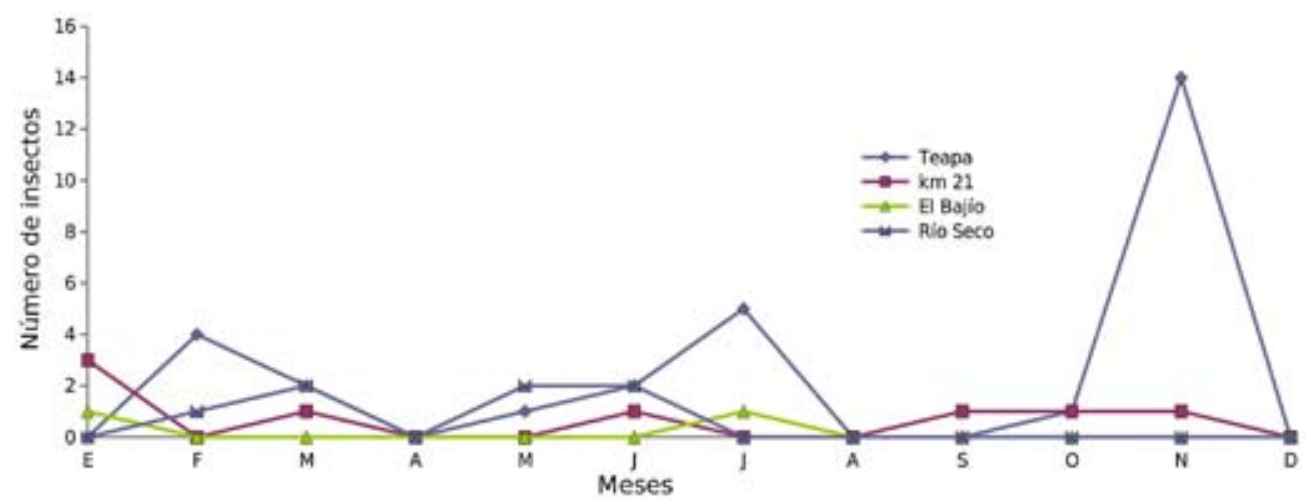

Figura 3. Fluctuación poblacional de platipódidos recolectados en cada una de las localidades estudiadas con trampas de luz.

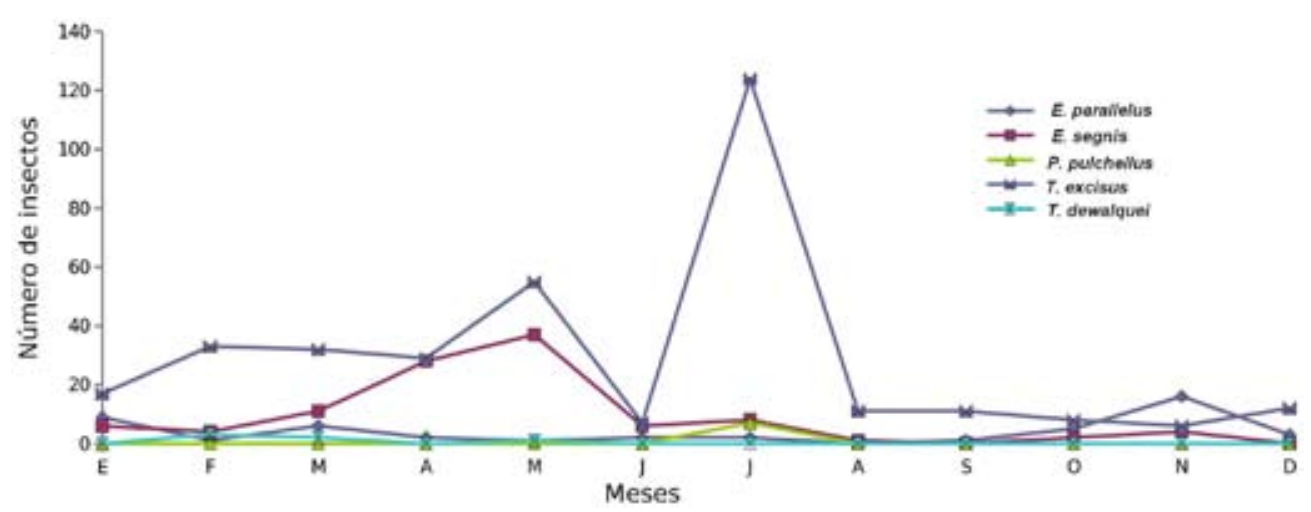

Figura 4. Fluctuación poblacional de las especies de platipódidos recolectados en cada una de las localidades estudiadas con los tres métodos captura.

\section{Plantas huéspedes y distribución de platipódidos}

1. Euplatypus parallelus (Fabricius), 1801. Se recolectaron 42 machos $(4.1 \mathrm{~mm}) \mathrm{y}$ seis hembras (4.6mm). Es la especie de Platypodinae más destructiva y de amplia distribución en África. Fue introducida a Angola, Camerún, Chad, Congo, Guinea Ecuatorial, Gabón, Chana, Guinea, Kenia, Nigeria, Isla Príncipe, Senegal, Sierra Leona, en el Sur de África se encuentra en Tanzania, Togo, Uganda, Zaire, se le registra en Australia y Madagascar. En el nuevo mundo se registra en las Antillas, Cuba, República Dominicana, Jamaica y Puerto Rico. En Norte América se tienen registros en California, Florida, Texas, México (Baja California Norte, Campeche, Chihuahua, Chiapas, Morelos, Nayarit, Nuevo León, Jalisco, Veracruz, Guerrero, Oaxaca, San Luis Potosí, Sonora, Sinaloa, Quin- 


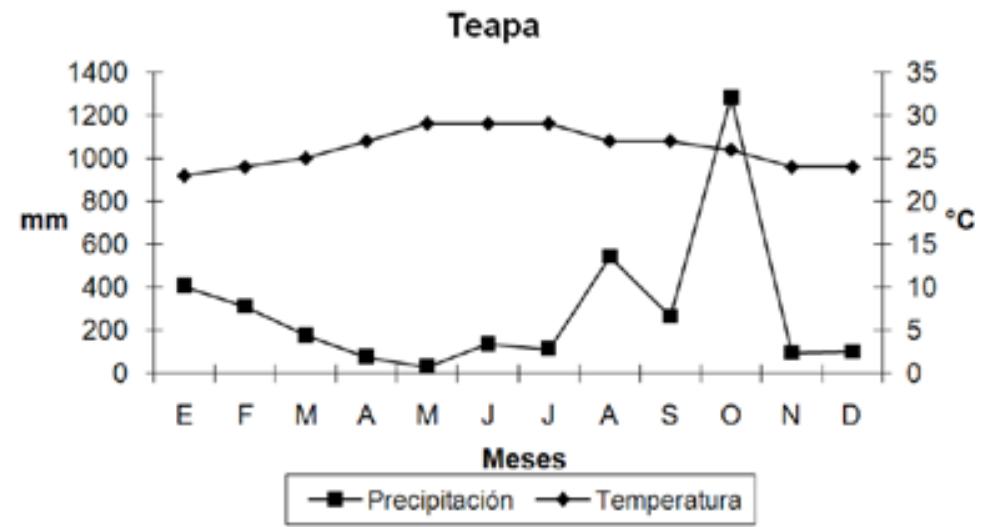

Figura 5. Promedios mensuales de precipitación $(\mathrm{mm})$ y temperatura $\left({ }^{\circ} \mathrm{C}\right)$ en el año 2007 en Teapa Tabasco.

\section{Cárdenas}

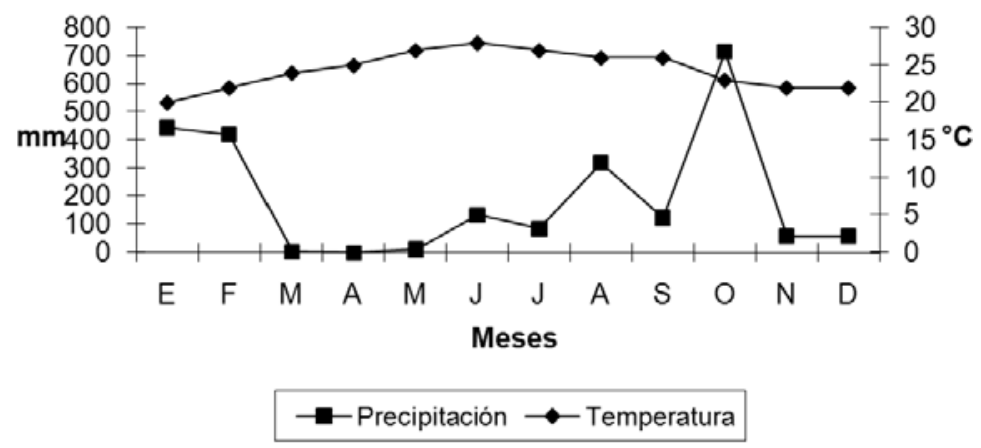

Figura 6. Promedios mensuales de precipitación $(\mathrm{mm})$ y temperatura $\left({ }^{\circ} \mathrm{C}\right)$ en el año 2007 en Cárdenas, Tabasco.

tana Roo, Tabasco, Tamaulipas y Yucatán), en Centro y Sudamérica. Huéspedes: Cocos nucifera, Ceiba sp., Ceiba aesculifolia Celtis laevigata, Mangifera indica, Acrocarpus sp., Pinus oocarpa, Theobroma cacao, Castillea costaricensis, Cedrella mexicana, Citrus sinensis, Astronium graveolens, Ficus nitida, F. elastica, F. continifolia, F. lyrata, F. retusa nitida, Ficus sp., Bursera sp., B. fagaroides, Delonix regia, Fraxinus udhei, Gymnopodium floribundum, Pouteria neglecta, Heliocarpus appendiculatus, Heliocarpus sp., Brosimium alicastrum, Metopium brownei, Aspidospermun megalocarpon, Croton nitens, Acacia dolychostachya, A. gaumeri, Lonchocarpus rugosus, Lysiloma baha- 
mensis, Gmelina arborea, Vitex guameri, Spondias mombin (Sharp \& Blandford 1895-1907, Equihua \& Atkinson 1987, Estrada \& Atkinson 1988, Wood \& Bright 1992b, Romero et al. 1997, Burgos-Solorio \& Equihua 2007).

2. Euplatypus segnis (Chapuis), 1865. Se recolectaron 92 machos $(3.6 \mathrm{~mm})$ y 15 hembras $(3.7 \mathrm{~mm})$. La distribución de esta especie está restringida al nuevo mundo; se le ha registrado en Antillas, Bolivia, Brasil, Colombia, Costa Rica, Guadalupe, Guatemala, Panamá, Perú, Venezuela y México (Chiapas, Jalisco, Morelos, San Luis Potosí, Tabasco y Veracruz). Huéspedes: Persea gratissima, Juglans sp., Mangifera indica, Bursera sp., B. fagaroides, Delonix regia, Ficus continifolia, F. elastica, F. lyrata, F. retusa nitida, Fraxinus udhei, Gymnopodium floribundum, Pouteria neglecta, Heliocarpus appendiculatus, Heliocarpus sp., Carya illnoensis (Sharp \& Blandford 1895-1907, Atkinson et al. 1986, Equihua \& Atkinson 1987, Estrada \& Atkinson 1988; Wood \& Bright 1992b, Romero et al. 1997, Burgos-Solorio \& Equihua 2007).

3. Platyscapulus pulchellus (Chapuis), 1865.Se recolectaron dos machos $(2.7 \mathrm{~mm})$ y cinco hembras $(3.0 \mathrm{~mm})$. Es una especie que también está sólo en América, en los siguientes países: Belice, Brasil, Costa Rica, El Salvador, Guatemala, Guyana, Honduras, Nicaragua, Surinam, Venezuela y México (Jalisco, Quintana Roo, Chiapas, Nayarit, Veracruz, Tabasco, Oaxaca y Campeche). Huésped: Gossypiospermum praecox, Brosimium alicastrum, Ficus sp., Croton pseudoniveus, Tabebuia sp.. Spondias mombin se cita como un nuevo huésped (Atkinson et al. 1986, Estrada \& Atkinson 1988; Wood \& Bright 1992b, Romero et al. 1997, Burgos-Solorio \& Equihua 2007).

4. Teloplatypus excisus (Chapuis), 1865. Se recolectaron 152 machos (2.8mm) y 194 hembras $(3.0 \mathrm{~mm})$. También es una especie americana y se le ha registrado en Antillas, Argentina, Brasil, Costa Rica, Guatemala, Guyana, Panamá, Paraguay, Puerto Rico, y México (Veracruz, Campeche, Morelos, Michoacán, Puebla, Guerrero, Jalisco, Veracruz, Chiapas, Tabasco). Huéspedes: Inga vera, Inga sp., Citrus sinensis, Dialium guianense, Lysiloma bahamensis, Lonchocarpus parviflorus, Pithecollobium dulce, P. manguense, Pterocarpus rohrii, Rhamnus capraefolia, Spondias mombin constituye un nuevo registro para este coleóptero (Atkinson et al. 1986, Estrada \& Atkinson 1988, Wood \& Bright 1992b, Romero et al. 1997, Burgos-Solorio \& Equihua 2007).

5. Tesserocerus dewalquei Chapuis 1865 . Se recolectaron tres machos $(5.6 \mathrm{~mm})$ $\mathrm{y}$ tres hembras $(5.6 \mathrm{~mm})$. De las cinco especies, ésta es la que presenta la distribución más restringida en México, y se cita para Costa Rica, El Salvador, Guatemala, Honduras, Nicaragua, Panamá, Argentina, Bolivia, Brasil, Colombia, Fr. Guiana, Paraguay, Perú, Surinam, Venezuela y México (Nayarit, Veracruz, Campeche, Oaxaca y Tabasco). Huéspedes: Caryocar nucifera, Hevea brasiliensis y Ocotea rodiaci (Wood \& Bright, 1992b, Estrada \& Atkinson 1988). 
Clave para la identificación de Platypodinae asociados al agroecosistema cacao en Tabasco, México. Las características utilizadas para la realización de esta clave fueron tomadas de la diagnosis de los géneros de platipódidos (Wood 1993). Debido a que este grupo de insectos presenta dimorfismo sexual marcado, se tomaron en cuenta las características de los élitros, abdomen y tórax de los machos por poseer las características más evidentes, además, por su forma de vida pueden ser capturados con facilidad. Aunado a la clave, se presentan fotografías de las especies que ayudan a su identificación (Figura 7).

1' Macho con un par de espinas visibles en el esternón abdominal 5, el declive del élitro es corto truncado con una ligera depresión en el ápice, ángulos ventrolaterales pobremente desarrollados, la interestría elitral del macho es usualmente carinada a partir de la mitad posterior del disco; $2.7 \mathrm{~mm}$ de longitud (Figura 7F) Platyscapulus pulchellus

1' Macho sin espinas visibles en el esternón abdominal 5 .................................2

2' (1') Declive elitral del macho con solamente un par de serraciones débiles en el margen ventrolateral, márgenes basales de los élitros usualmente armados por pequeñas espinas o gránulos en la interestría 1,3 y $5 ; 2.8 \mathrm{~mm}$ de longitud (Figura 7B) ................................................................Teloplatypus excisus

2' Especies con declive elitral armado con fuertes procesos espinales.................3

3' (2') Pronoto formando un cuadrado en vista dorsal, los ángulos ventrolaterales del declive del macho se extiende en un par de procesos que exceden el ápice sutural armados con dientes .......................................................Euplatypus 4

3' Pronoto redondeado en los márgenes laterales, declive elitral fuertemente truncado, armado con fuertes espinas en la parte distal, márgenes ventrolaterales armados con dos espinas prominentes; $5.6 \mathrm{~mm}$ de longitud (Figura $7 \mathrm{~J})$ Tesserocerus dewalquei

4' (3) Declive elitral angosto hacia la región apical en vista dorsal, con procesos ventrolaterales proyectados en puntas bien definidas, cada proceso armados con tres dientes bien definidos; $3.6 \mathrm{~mm}$ de longitud (Figura $7 \mathrm{H})$ Euplatypus segnis

4' Declive elitral más ancho hacia la región apical en vista dorsal, con procesos ventrolaterales proyectados en punta no tan marcadas (roma), cada proceso armado con tres dientes en forma de gránulos; $4.1 \mathrm{~mm}$ de longitud (Figura 7D) Euplatypus parallelus

\section{DISCUSIÓN}

Se reportan cinco de las seis especies de platipódidos registradas para Tabasco (Sharp \& Blandford 1895-1907), lo que representa el 12.5\% del total de las especies (40 especies) registradas para México (Romero et al. 1997) por lo que es una cifra con- 

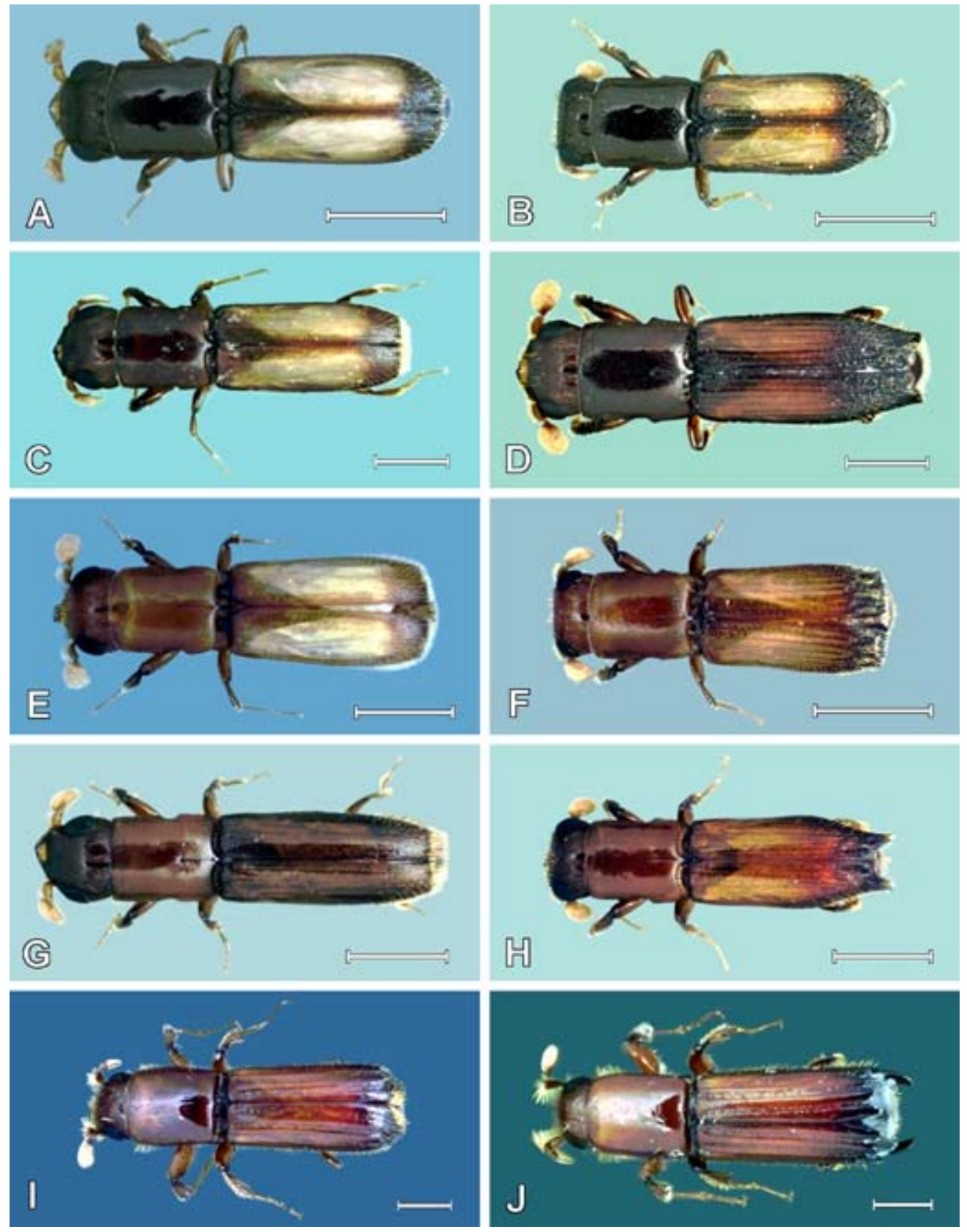

Figura 7. Escala: 1mm, A. Teloplatypus excisus (†), B. T. excisus (ठ); C. Euplatypus parallelus ( $($ ), D. E. parallelus (ठ); E. Platyscapulus pulchellus (), F. P. pulchellus (ठ), G. Euplatypus segnis (ㅇ), H. E. segnis (ठ); I. Tesseroserus dawalquei (ㅇ), J. T. dewalquei (ð). 
siderable si tomamos en cuenta que el estudio fue enfocado al agroecosistema cacao; estos resultados coinciden con el número de especies (5 especies) reportadas por Estrada \& Atkinson (1988) en un área donde se incluyó tierras agrícolas, acahuales y restos de selva; además de compartir cuatro de las cinco especies recolectadas en este estudio. De igual forma este estudio comparte cinco especies de las 14 registrada en el trabajo realizado por Atkinson \& Equihua (1986) en un área de bosque y tierra agrícolas de Veracruz y Oaxaca.

La trampa de alcohol fue el método de captura más eficiente para platipódidos ya que al igual que los escolítidos guían su vuelo hacia áreas de liberación de oleorresinas volátiles, terpenos e hidrocarburos, alcoholes (el alcohol etílico es un producto similar al que produce la madera como resultado de la fermentación anaerobia) u otras substancias secretadas por los tejidos de las plantas huéspedes que están moribundas o recién cortadas (Rudinsky 1962, Wood 1982), por lo cual este método de captura facilita la recolecta de estos insectos y se recomienda ampliamente para cuestiones de monitoreo de las poblaciones de insectos con hábitos similares, actualmente se utiliza el alcohol etílico como atrayente de trampas como una de las alternativas para el control de la broca del café (Hypothenemus hampei Ferrari) en México y en América del sur.

La fluctuación poblacional de los platipódidos, está influenciada por la disponibilidad del recurso; como es el alimento, depredadores, competidores y factores ambientales (temperatura, humedad y lluvia) los cuales en conjunto determinan la distribución y abundancia de platipódidos a nivel local y regional, algo parecido a lo que ocurre con los escolítidos (Wood 1982), dado que son insectos con hábitos similares en esta misma región (Pérez et al.2009), a pesar de ello ambos grupos pueden convivir y compartir el recurso disponible en un mismo ambiente donde las poblaciones mejores adaptadas con ciertas estrategias (selección de hospederos, hábitos de alimentación, reproducción, supervivencia, depredadores, etc.,) utilizan el recurso presentando poblaciones más elevadas. Además se registro en este estudio que las poblaciones de platipódidos se encuentran en al menos tres de los sitios estudiados con poblaciones bajas y compartiendo huéspedes; tal es el caso de E. parallelus que comparte Spondias mombin y Theobroma cacao con especies de escolítidos (Xyleborus affinis Eichhoff, $X$. ferrugineus(Fabricius) y $X$. volvulus (Fabricius)) y con platipódidos (P. pulchellus y T. excisus)) a S. mombin (Pérez et al.2009), aunado al limitado recurso de sus hospederos ya que $S$. mombin se encuentra como árbol de sombra en el agroecosistema cacao y en bajas densidades lo que da paso a que sus poblaciones se mantengan en niveles bajos a diferencia de los observado en Teapa donde existe una mayor disponibilidad de recursos ( $S$. mombin) y a juzgar por su abundancia $T$. excisus y E. segnis han utilizado el recurso y aprovechado las condiciones ambientales que les han permitido sobresalir con poblaciones relativamente más abundantes que las otras especies de platipódidos, de tal forma que presentan picos poblacionales 
marcados en mayo y julio las cuales influyen en la fluctuación poblacional observada en las figuras 1,2 y 4.

La máxima abundancia de las poblaciones de platipódidos en los sitios estudiados esta relacionada probablemente a la disponibilidad de los recursos vegetales, la humedad y las temperaturas adecuadas que propician el establecimiento y desarrollo de los hongos asociados que le sirven de alimento, dando como resultado que sus poblaciones alcancen su máximo desarrollo cuando las condiciones adecuadas se presentan [en este estudio se registró al inicio de la temporada de lluvia en julio (en la mayoría de los sitios estudiados) y noviembre (trampas de luz en Teapa) de acuerdo a las capturas de estos insectos]. Aunque cabe señalar que durante los demás meses las especies mantiene sus poblaciones en niveles bajos a través del año, sin llegar a estar ausente por completo, lo que indica que tienen periodos de vuelo durante todo el año pero de manera restringida, algo similar a lo registrado para los escolítidos asociados al agroecosistema cacao en esta misma área (Pérez et al. 2009).

La amplia distribución de $E$. parallelus, está influenciada por la gama de huéspedes que presenta; a diferencia de $T$. dewalquei que se restringe a sólo tres especies de huéspedes ( $C$. nucifera, $H$. brasiliensis y $O$. rodiaci), sin embargo hacen falta estudios detallados sobre la biología de las especies de platipódidos en diferentes ambientes de México.

Esta información puede coadyuvar como herramienta para un futuro manejo dentro del agroecosistema cacao, sobre todo en las especies de importancia económica como E. parallelus que sus daños reducen el valor comercial de la madera (el agroecosistema cacao presenta una diversidad amplia de especies maderables y frutales), además está directamente asociada al T. cacao. Algunas de las estrategias que pueden ser sugeridas y aplicadas para el manejo de estos insectos pueden ser el derribo y troceado de árboles enfermos que sirven de hospederos a estos insectos con la finalidad de disminuir la humedad y acelerar el proceso de descomposición del material vegetal, aunque es importante no almacenar material vegetal dentro del ecosistema dado que esto puede favorecer el incremento de las poblaciones de platipódidos, además cabe señalar que de manera tradicional los agricultores utilizan el material vegetal (leña para cocinar sus alimentos) generado en el agroecosistema cacao, lo cual contribuye de manera directa a mantener bajas las poblaciones de estos insectos al regularse la disponibilidad de alimento en el sistema, lo cual puede ayudar a explicar las bajas poblaciones en algunos sitios estudiados (El Bajío y Río seco son plantaciones de tras patio donde el agricultor está en contacto directo y utilizando los recursos vegetales de manera constante) a diferencia de las otras plantaciones que se encuentran a una distancia mayor de las poblaciones humanas, lo que dificulta al agricultor el traslado de los recursos vegetales. Para cuestiones de manejo se sugiere consultar algunas de las estrategias utilizadas para el combate de los escolítidos en zonas tropicales descritas por Cibrián et al. (1995). 


\section{LITERATURA CITADA}

Arnnet, R. H. 1973. The beetles of the United States (A manual for identification). The American Entomological Institute. Londonville, New York 1029-1030 p.

Atkinson, T. H. \& A. Equihua M. 1985. Lista comentada de los coleópteros Scolytidae y Platypodidae del valle de México. Folia Entomológica Mexicana, 65: 63-108.

Atkinson, T. H., E. Martínez F., E. Saucedo C. \& A. Burgos S. 1986. Los Scolytidae y Platypodidae (Coleoptera). Asociados a Selva baja caducifolia y comunidades derivadas en el estado de Morelos. Folia Entomológica Mexicana. 69: 41-82.

Atkinson, T. H. \& A. Equihua M. 1986. Biology of Bark and Ambrosia Beetles (Coleoptera: Scolytidae and Platypodidae) of a Tropical Rain Forest in Southeastern Mexico with an Annotated Checklist of species. Annals of the Entomological Society of America. 79: 414-423.

Atkinson, T. H. \& A Equihua M. 1988. Notas sobre biología y distribución de Scolytidae y Platypodidae (Coleoptera) de México y Centroamérica. Folia Entomológica Mexicana, 76: 83-105.

Bright, D. E. \& R. E. Skidmore. 1997. A Catalog of Scolytidae and Platypodidae (Coleoptera), Suplement 1 (1990-1994). NRC press. Ottawa. 368 p.

Bright, D. E. \& R. E. Skidmore. 2002. A Catalog of Scolytidae and Platypodidae (Coleoptera), Suplement 2 (1995-1999). NRC press. Ottawa. 523 p.

Burgos-Solorio, A. \& M. A. Equihua. 2007. Platypodidae y Scolytidae (Coleoptera) de Jalisco, México. Dugesiana, 14: 59-82.

Bustamante, O. F. \& T. H. Atkinson. 1984. Biología del barrenador de las ramas del peral Corthylus fuscus Blandford (Coleoptera: Scolytidae), en el norte del Estado de Morelos. Folia Entomológica Mexicana, 60: 83-101.

Equihua, M. A., T. H. Atkinson \& E. Lott. 1984. Scolytidae y Platypodidae (Coleoptera) de la estación de biología Chamela, Jalisco. Agrociencia, 57: 179-193.

Equihua, M. A. 1985. Nuevos registros de localidades y huéspedes de coleópteros Platypodidae Americanos. Folia Entomológica Mexicana, 66: 143-144.

Equihua, M. A. \& T. H. Atkinson. 1987. Catálogo de Platypodidae (Coleoptera) de norte y Centroamérica. Folia Entomológica Mexicana, 72: 5-31.

Estrada, V.A. \& T.H. Atkinson. 1988. Scolytidae y Platypodidae (Coleoptera) de Escárcega, Campeche, México. Biogeografía, biología, importancia económica y una lista comentada de especies. Anales del Instituto de Biología UNAM Serie Zoológica, 58: 199-220.

Cibrián, T. D., M. J. T. Méndez, B. R. Campos, H. O. Yates III \& L. J. Flores. 1995. Insectos Forestales de México/Forest Insects of Mexico. Universidad Autónoma Chapingo. México. 453 p.

INEGI (Instituto Nacional de Estadística Geografía e Informática). 2005. Cuaderno estadístico Municipal, Cárdenas, Tabasco. México. INEGI. 187 p.

INEGI (Instituto Nacional de Estadística Geografía e Informática). 2006. Cuaderno estadístico Municipal, Teapa, Tabasco. México. INEGI. 211 p.

Iturre, M. \& E. Darchuck. 1996. Registro de escolítidos relacionados al género Eucalyptus en Santiago del Estero. Quebracho, 4: 11-16.

Pérez, De la C. M., M. A. Equihua, N. J. Romero, S. S. Sánchez, \& L. E. García. 2009. Diversidad, fluctuación poblacional y plantas huésped de escolítinos (Coleoptera: Curculionidae) asociados con el agroecosistema cacao en Tabasco, México. Revista Mexicana de Biodiversidad, 80: 779-791.

Romero, N. J., R. S. Anaya, M. A. Equihua \& G. H. Mejía. 1997. Lista de Scolytidae y Platypodidae de México (Insecta: Coleoptera). Acta Zoológica Mexicana (n. s.), 70: 35-53.

Rudinsky L. A. 1962. Ecology of Scolytidae. Annual Review of Entomology, 7: 327-348.

Schedl, K. E. 1940. Scolytidae, Coptonotidae y Platypodidae mexicanos. Anales de la Escuela Nacional de Ciencias Biológicas, (Instituto Politécnico Nacional), 1: 317-378. 
Sharp, D. \& W. F. H. Blandford; K. Jordan. Insecta. Coleoptera. Rhyncophora. Volumen IV, Part 6. [London: Published for the editors by R.H. Porter]: 1895-1907.

Wood, S. L. 1982. The bark and ambrosia beetles of North and Central America (Coleoptera: Scolytidae), a Taxonomic Monograph. Great Basin Naturalist Memoirs, 6: 1-1359.

Wood, S. L. 1993. Revision of the genera of Platypodidae (Coleoptera). Great Basin Naturalist Memoirs, 53: 259-281.

Wood, S. L. \& D. E. Bright. 1992a. A catalog of Scolytidae and Platypodidae (Coleoptera), Part 2: Taxonomic Index. Vol. A. Great Basin Naturalist Memoirs, 13: 1-833.

Wood, S. L. \& D. E. Bright. 1992b. A catalog of Scolytidae and Platypodidae (Coleoptera), Part 2: Taxonomic Index. Vol. B. Great Basin Naturalist Memoirs, 13: 835-1553. 